\title{
Ebselen Reacts with SARS Coronavirus-2 Main Protease Crystals
}

2 Tek Narsingh Malla ${ }^{1}$, Suraj Pandey ${ }^{1}$, Ishwor Poudyal ${ }^{1}$, Denisse Feliz ${ }^{2}$, Moraima Noda $^{2}$,

3 George Phillips ${ }^{3}$, Emina Stojkovic ${ }^{2}$, Marius Schmidt ${ }^{1}$

4 University of Wisconsin-Milwaukee, Milwaukee, WI

5 Northeastern Illinois University, Chicago, IL

6 Rice University, Houston, TX

Abstract. The SARS coronavirus 2 main protease 3CLpro tailor cuts various essential virus proteins out of long poly-protein translated from the virus RNA. If the 3CLpro is inhibited, the functional virus proteins cannot form and the virus cannot replicate and assemble. Any compound that inhibits the 3CLpro is therefore a potential drug to end the pandemic. Here we show that the diffraction power of 3CLpro crystals is effectively destroyed by Ebselen. It appears that Ebselen may be a widely available, relatively cost effective way to eliminate the SARS coronavirus 2.

Introduction.

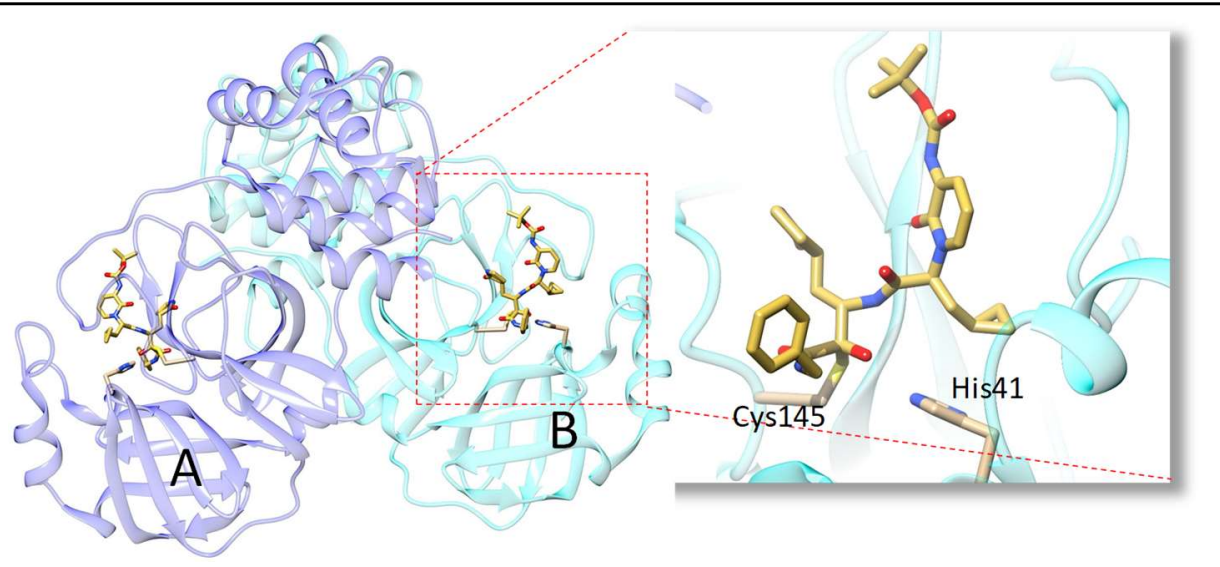

Figure 1. SARS CoV-2 3CLpro in its functional, dimeric form ${ }^{1}$. The two subunits A and B are shown in dark and light blue. An $\alpha$-ketoamide inhibitor is bound to the active site (red box, enlarged). The His-41/Cys-145 catalytic dyad is marked.

During the COVID-19 pandemic in the last half year, a large body of literature became available to understand and combat the SARS coronavirus-2 (CoV-2). Due to its importance for virus protein maturation the SARS CoV-2 main protease (Mpro), also called 3CLpro, became a major drug target. In its functional form the 3CLpro is a homo dimer with His/Cys dyads (His-41 and Cys145 ) in the active centers ${ }^{1}$. The structure of the CoV-2 3CLpro has been solved recently (Fig. 1) guided by high similarity to other coronavirus $3 \mathrm{CLpros}^{1}$. A large number $(>500)$ of SARS CoV-2 3CLpro structures were recently deposited in the protein data bank mainly following a fragment screening study at the synchrotron Diamond near Oxford, England. Binding of fragments not only to the active center was observed. They all constitute a database of potential drugs to target the 3CLpro. Apart from the fragments, the most promising compounds are the $\alpha$-ketoamides (Fig. 1) which bind tightly to the $3 \mathrm{CLpro}^{1-3}$. As they are complicated to synthesize they carry a hefty price 
tag (companies ask for 14,000 dollars/g as recently inquired by one of the authors). A less expensive, but also less known compound that binds to the 3CLpro is Ebselen ${ }^{4}$. Ebselen is a selenium compound (Scheme 1) currently tested for a number of diseases such as bipolar disorder and hearing $\operatorname{loss}^{4}$. Selenium is an essential metal, but toxic in higher doses. Ebselen has been shown to bind strongly to the CoV-2 3CLpro ${ }^{4}$, but the structure of the complex is unknown. Here we show what happens when Ebselen is added to 3CLpro crystals.

Methods.

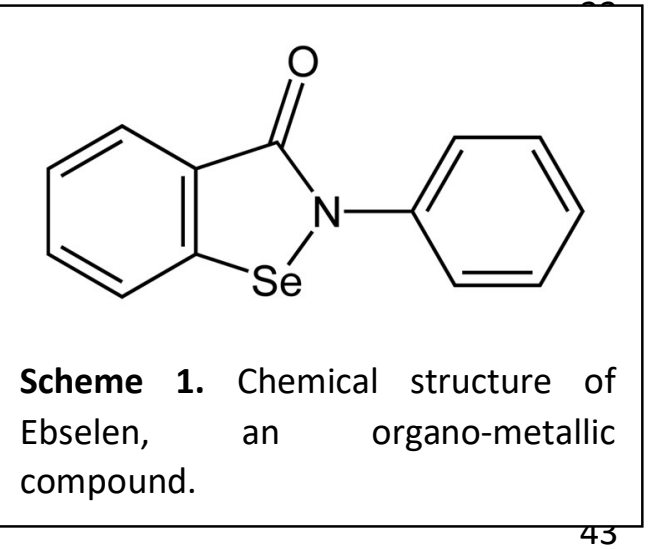

Expression. The CoV-2 3CLpro sequence was synthetized (GenScript) for optimized expression in E. coli according to sequence information published previously ${ }^{1}$. In short, the N-terminus of 3CLpro is fused to glutathione-S-transferase (GST). It further has a 6-His tag at the c-terminus. The N-terminal GST will be autocatalytically cleaved off after expression due to an engineered 3CLpro cleavage sequence. The His tag can cleaved off by a PreCission Protease. Overexpression and protein purification protocols were modified from previous reports. E. coli were grown to $0.8 \mathrm{OD}_{600}$ at $37^{\circ}$ in terrific broth. Expression was induced by $1 \mathrm{mmol} / \mathrm{L}$ IPTG at $25^{\circ} \mathrm{C}$. After $3 \mathrm{~h}$ of expression, the culture was induced a second time ( $1 \mathrm{mmol} / \mathrm{L} \mathrm{IPTG})$, and shaken overnight at $20^{\circ} \mathrm{C}$. The yield is about $80 \mathrm{mg}$ for a $6 \mathrm{~L}$ culture. Cells were resuspended in lysis buffer $(20 \mathrm{mM}$ Tris Base, 150 $\mathrm{mmol} / \mathrm{L} \mathrm{NaCl}, \mathrm{pH} 7.8$.). After lysis of the bacterial cells, debris was centrifuged at 50,000 $\mathrm{g}$ for 1 hour. The lysate was let stand at room temperature for at least $3 \mathrm{~h}$ (overnight is also possible). After this, the lysate was pumped through a column containing $15 \mathrm{~mL}$ of Talon Cobalt resin 50 (TAKARA). The resin was washed without using imidazole using a wash cycle consisting of low

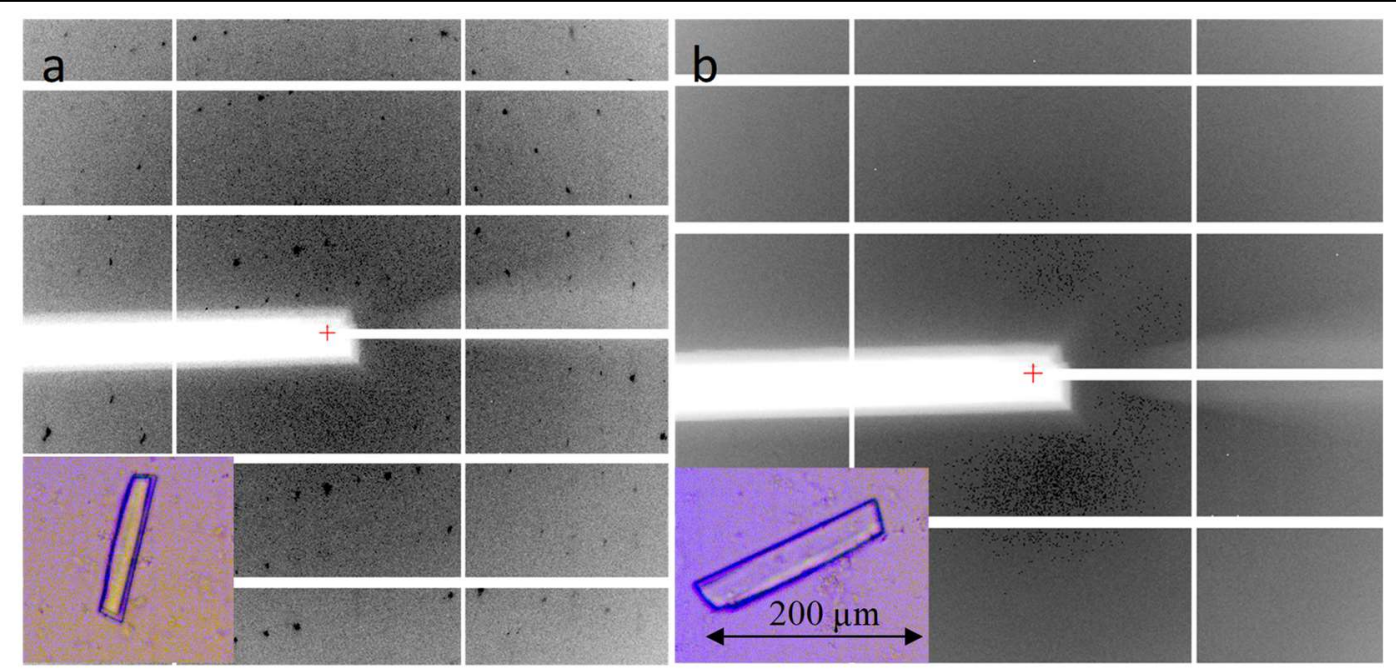

Figure 2. Low resolution $(\sim 3.5 \AA$ in the corner) diffraction patterns of SARS CoV2 3CLpro. (a) no ligand, (b) with Ebselen. Inserts: crystal images w/o and w Ebselen, respectively 


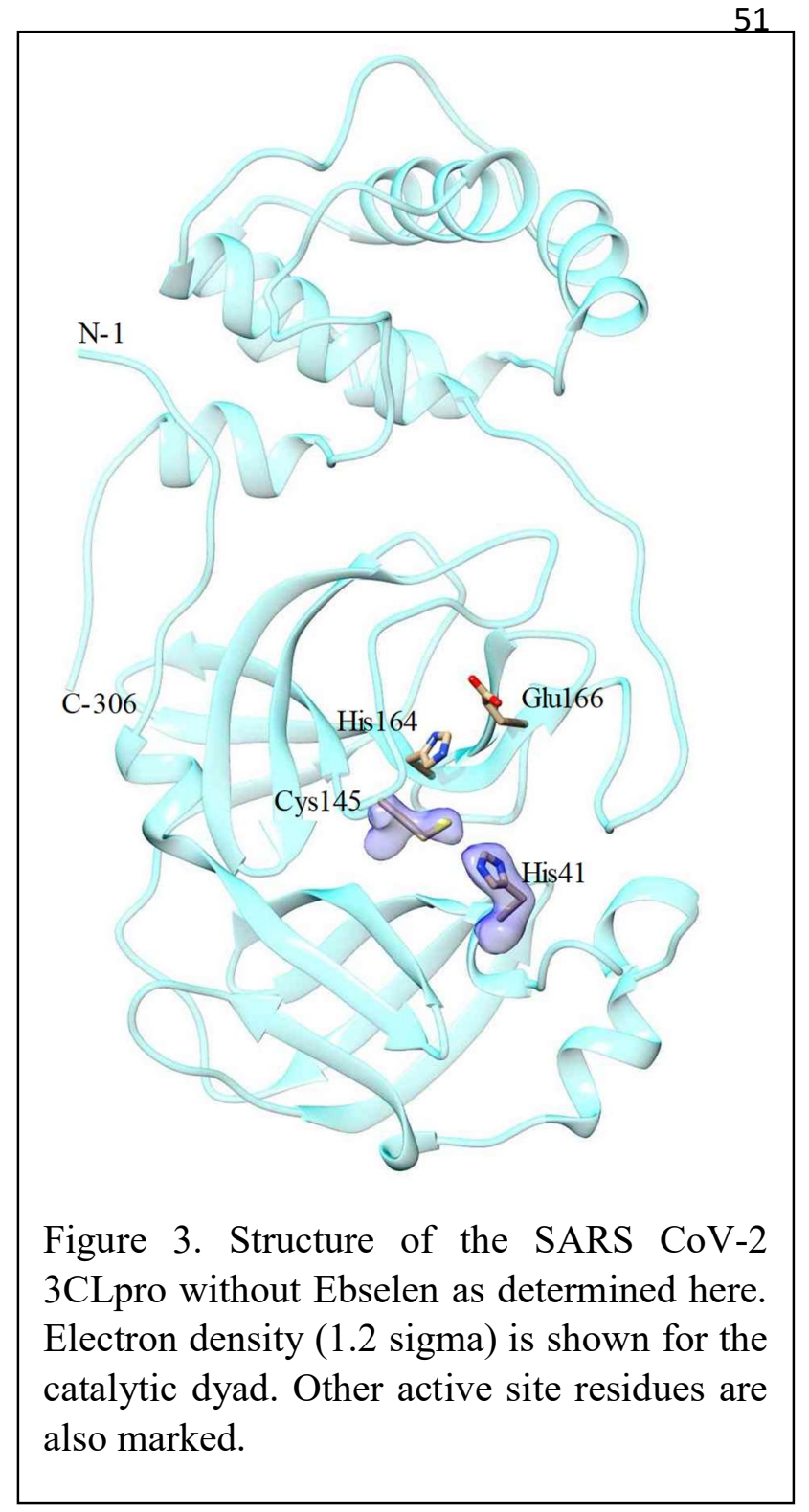

81 salt $(20 \mathrm{mmol} / \mathrm{L}$ Tris Base, $50 \mathrm{mmol} / \mathrm{L} \mathrm{NaCl}$, $\mathrm{pH}$ 7.8), high salt $(20 \mathrm{mmol} / \mathrm{L}$ Tris Base, 1 $\mathrm{mol} / \mathrm{L} \mathrm{NaCl}, \mathrm{pH} \mathrm{7.8)}$ and low salt (as above) solutions (about 20 column volumes each). After the wash cycle was completed, the column was let stand for an additional $2 \mathrm{~h}$ at room temperature followed by another wash cycle. The final product was eluted by 300 $\mathrm{mmol} / \mathrm{L}$ imidazole, dialyzed immediately in $20 \mathrm{mmol} / \mathrm{L}$ Tris base, $150 \mathrm{mmol} / \mathrm{L} \mathrm{NaCl}, 0.1$ $\mathrm{mmol} / \mathrm{L}$ dithiotreitol (DTT), $\mathrm{pH} \mathrm{7.8,} \mathrm{and}$ concentrated to $20 \mathrm{mg} / \mathrm{mL}$. Note, the cterminal 6-His tag was not cleaved off. Due to this one step purification protocol, only $24 \mathrm{~h}$ are required from cell lysis to the pure 3CLpro product. The product is within 1.7 Da of the theoretical molecular weight as determined by mass spectroscopy.

Crystallization. The concentrated 3CLpro (with the His-tag left on) was diluted to 4 $\mathrm{mg} / \mathrm{mL} .100 \mu \mathrm{L}$ of the diluted 3CLpro was mixed (1:1) in batch mode with the same amount of $25 \%$ PEG 3350, Bis-Tris 100 $\mathrm{mmol} / \mathrm{L}, \mathrm{pH}$ 6.5. A few days later, nicely shaped crystals with dimensions of about 200 x 30 x $30 \mu^{3}$ were appearing. Crystals were soaked in mother liquor by adding Ebselen in powder form as Ebselen is not very soluble in water. Enough Ebselen that would otherwise produce a $50 \mathrm{mmol} / \mathrm{L}$ solution was added. After 2 days of soaking, the crystals completely maintained their morphology (Fig. 2b).

Data Collection and Structure determination. The crystals were mounted in Mitegen microloops (30 - $50 \mu \mathrm{m}$ ) and directly frozen in pucks suspended in liquid nitrogen for automated (robotic) data collection. The dewar with the pucks were driven to the Advanced Photon Source for robotic data collection at Sector-19 (Structural Biology Center, SBC, beamline 19-ID-D). Data collection was fully remote due to restriction of the COVID-19 pandemic. Fig. 2 shows a comparison of diffraction patterns collected from untreated crystals (Fig. 2a), and crystals treated with the Ebselen (Fig. 2b). As the untreated crystals diffracted beyond $2 \AA$, the treated crystals did not show any Bragg reflections whatsoever, even at lowest resolution. They are completely amorphous, despite the nice crystal-like shape. Accordingly, the structure of only the untreated 3CLpro can be solved. A dataset to $2.2 \AA$ was collected $\left(0.5^{\circ}\right.$ rotation and $0.3 \mathrm{~s}$ exposure per detector readout for a total 
of $180^{\circ}$ ). Data was processed with HKL $3000^{5}$. Data statistics in shown in Tab. 1. Initially the spacegroup was found to be $\mathrm{C} 2$ (monoclinic centered). It was further determined by the CCP4 program ${ }^{6}$ pointless that spacegroup $\mathrm{I} 2$ (monoclinic body centered) is more suitable, in accordance with published results ${ }^{7}$. The 3 CLpro structure with pdb access code $6 \mathrm{WQF}^{7}$ was used as initial model. Molecular replacement was not necessary. The model fits immediately and can be used for refinement. Refinement was done using $\operatorname{refmac}^{8}$ (version 5.8.0238). The structure is shown in Fig. 3. Its mean square deviation from model $6 \mathrm{WQF}$ is about $0.4 \AA$ with $0.5 \AA$ standard deviation. This means that the 3CLpro cryo structure with the His-tag and the room temperature structure are identical within the resolution limit. The structure of the His-tag could not be determined. There is, however, a large crystal cavity near the C-terminus that might accommodate (a very disordered) 6 histidine structure.

Results and Discussion. A very important result is that c-terminal PreCission His-tag cleavage is not required to obtain 3CLpro crystals. This speeds up purification to the extent that now gram sized 3CLpro preparations can be obtained in a relatively short period of time. This is a prerequisite for mix-and-inject approaches at free electron lasers with gas-dynamic-virtual-nozzle type mixing injectors ${ }^{9,10}$. At XFELs low concentrations of Ebselen can be mixed with the crystalline slurry which is quickly injected into the X-ray beam. There is hope that crystal decomposition is slower than the delay between mixing and injection. Then, the structure of the Ebselen-3CLpro complex may be determined. In addition, microcrystals also tend to be unusually flexible and may survive large unit cell changes ${ }^{11}$, which might make these experiments even more conceivable.

Since Ebselen has this strong effect on 3CLpro crystals, it a clear indication for the tight affinity of this compound to the 3CLpro. Binding of Ebselen to the catalytic dyad has been already identified by mass-spectroscopy and published earlier ${ }^{4}$. However, also after the experiments reported here, the 3CLpro-Ebselen structure remains elusive. As Ebselen is a potent drug (and it is not too toxic even in higher concentrations ${ }^{4}$ ), it has to be seen whether it can be used as an effective weapon against the SARS CoV-2, and maybe all other pathogenic coronaviruses. Whether it is suitable for a drug must be determined by physicians in a clinical setting perhaps in combination with a SARS CoV-2 RNA dependent RNA polymerase inhibitor such as remdesivir ${ }^{12}$. As crystallographers and structural biologists, though, we can deliver evidence for a potential mechanism as shown recently by others for the $\alpha$-ketoamides ${ }^{1,2}$ and also remdesivir ${ }^{12}$.

This manuscript is published without delay on BioRxiv to disseminate rapid methods for preparation, purification and structure determination of 3CLpro to a wide community. In addition, this manuscript intends to alert and increase awareness of the potential of Ebselen as a 3CLpro inhibitor and its value perhaps in combination, as the authors are not aware that anything is reported about it in the public press. The coordinates of the $100 \mathrm{~K} 3 \mathrm{CL}$ pro structure as determined here are available on request.

Acknowledgement. This work is supported by NSF grant STC 1727290 (BioXFEL) and NSF grant RAPID 2030466. Results shown in this report are derived from work performed at Argnonne National Laboratory (ANL), Structural Biology Center (SBC) at the Advanced Photon Source (APS), under U.S. Department of Energy, Office of Biological and Environmental Research 
133 contract DE-AC02-06CH11357. The authors thank Darren A. Sherrell for setting up the 134 experiment at the beamline.

135

136

Table. 1 Data collection and refinement statistics*

137

\begin{tabular}{|l|l|}
\hline & SARS CoV-2 3CLpro \\
\hline Beamline & $19-$ ID-D, APS \\
\hline Resolution & $2.2 \AA$ \\
\hline Temperature & $110 \mathrm{~K}$ \\
\hline Space group & $\mathrm{I} 2$ \\
\hline Unit-cell parameters $\left({ }^{\circ}\right)$ & $\begin{array}{l}\mathrm{a}=44.7 \AA \mathrm{b}=53.4 \AA \mathrm{c}=112.3 \AA \\
\alpha=90^{\circ} \beta=100.0^{\circ} \gamma=90^{\circ}\end{array}$ \\
\hline No of unique reflections & 9426 \\
\hline Redundancy & $3.1(1.9)$ \\
\hline Completeness $(\%)$ & 70.0 \\
\hline CC1/2 (\%) & $99.8(47.6)$ \\
\hline $\mathrm{R}_{\text {merge }}(\%)$ & $7.3(76.5)$ \\
\hline & \\
\hline Refinement & \\
\hline $\mathrm{R}_{\text {cryst }} / \mathrm{R}_{\text {free }}(\%)$ & $19.1 / 26.3$ \\
\hline RMSD to 6WQF & $0.39+/-0.53 \AA$ \\
\hline
\end{tabular}

\footnotetext{
*last resolution shell in brackets
} 


\section{References.}

1401 Zhang, L. L. et al. Crystal structure of SARS-CoV-2 main protease provides a basis for design of improved alpha-ketoamide inhibitors. Science 368, 409-+. Enterovirus Replication: Structure-Based Design, Synthesis, and Activity Assessment.

1453 Dai, W. et al. Structure-based design of antiviral drug candidates targeting the SARS-

1495 Minor, W., Cymborowski, M., Otwinowski, Z. \& Chruszcz, M. HKL-3000: the CoV-2 main protease. Science 368, 1331-1335, PMC7179937.

4 Jin, Z. M. et al. Structure of M-pro from SARS-CoV-2 and discovery of its inhibitors. Nature 582, 289-+. integration of data reduction and structure solution - from diffraction images to an initial model in minutes. Acta Crystallographica Section D-Structural Biology 62, 859-866.

6 Winn, M. D. et al. Overview of the CCP4 suite and current developments. Acta Crystallogr D 67, 235-242.

7 Kneller, D. W. et al. Structural plasticity of SARS-CoV-2 3CL M-pro active site cavity revealed by room temperature X-ray crystallography. Nat Commun 11.

8 Murshudov, G. N. et al. REFMAC5 for the refinement of macromolecular crystal structures. Acta crystallographica. Section D, Biological crystallography 67, 355-367, 3069751.

9 Olmos, J. L., Jr. et al. Enzyme intermediates captured "on the fly" by mix-and-inject serial crystallography. BMC Biol 16, 59, PMC5977757.

10 Calvey, G. D., Katz, A. M. \& Pollack, L. Microfluidic Mixing Injector Holder Enables Routine Structural Enzymology Measurements with Mix-and-Inject Serial Crystallography Using X-ray Free Electron Lasers. Anal Chem 91, 7139-7144.

11 Stagno, J. R. et al. Structures of riboswitch RNA reaction states by mix-and-inject XFEL serial crystallography. Nature 541, 242-246. from SARS-CoV-2 by remdesivir. Science 368, 1499-+. 